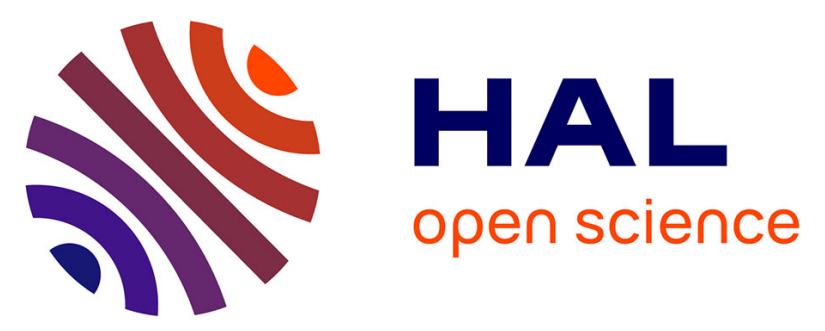

\title{
Capillary force on a micrometric sphere trapped at a fluid interface exhibiting arbitrary curvature gradients
} Christophe Blanc, Denys Fedorenko, Michel Gross, Martin In, Manouk Abkarian, Mohamed Amine Gharbi, Jean-Baptiste Fournier, Paolo Galatola, Maurizio Nobili

\section{To cite this version:}

Christophe Blanc, Denys Fedorenko, Michel Gross, Martin In, Manouk Abkarian, et al.. Capillary force on a micrometric sphere trapped at a fluid interface exhibiting arbitrary curvature gradients. Physical Review Letters, 2013, 111, pp.058302. 10.1103/PhysRevLett.111.058302 . hal-00840804

\section{HAL Id: hal-00840804 https://hal.science/hal-00840804}

Submitted on 4 Jun 2021

HAL is a multi-disciplinary open access archive for the deposit and dissemination of scientific research documents, whether they are published or not. The documents may come from teaching and research institutions in France or abroad, or from public or private research centers.
L'archive ouverte pluridisciplinaire HAL, est destinée au dépôt et à la diffusion de documents scientifiques de niveau recherche, publiés ou non, émanant des établissements d'enseignement et de recherche français ou étrangers, des laboratoires publics ou privés. 
See discussions, stats, and author profiles for this publication at: https://www.researchgate.net/publication/255972431

\section{Capillary Force on a Micrometric Sphere Trapped at a Fluid Interface Exhibiting Arbitrary Curvature Gradients}

Article in Physical Review Letters · August 2013

DOI: 10.1103/PhysRevLett.111.058302· Source: PubMed

CITATIONS

33

9 authors, including:

Christophe Blanc

Université de Montpellier

119 PUBLICATIONS 1,922 CITATIONS

SEE PROFILE

Martin In

Université de Montpellier

69 PUBLICATIONS 1,773 CITATIONS

SEE PROFILE

Some of the authors of this publication are also working on these related projects:

Holography View project

Project Brownian and active motion of partially wetted particles View project
READS

79

Michel Gross

Université de Montpellier

204 PUBLICATIONS 6,752 CITATIONS

SEE PROFILE

Mohamed Amine Gharbi

University of Massachusetts Boston

33 PUBLICATIONS 536 CITATIONS

SEE PROFILE 


\title{
Capillary Force on a Micrometric Sphere Trapped at a Fluid Interface Exhibiting Arbitrary Curvature Gradients
}

\author{
Christophe Blanc, ${ }^{1,2}$ Denys Fedorenko, ${ }^{1,2}$ Michel Gross, ${ }^{1,2}$ Martin In, ${ }^{1,2}$ Manouk Abkarian, ${ }^{1,2}$ Mohamed Amine Gharbi, ${ }^{1,2}$ \\ Jean-Baptiste Fournier, ${ }^{3}$ Paolo Galatola, ${ }^{3}$ and Maurizio Nobili ${ }^{1,2}$ \\ ${ }^{1}$ Université Montpellier 2, Laboratoire Charles Coulomb, UMR 5521, F-34095 Montpellier Cedex 5, France \\ ${ }^{2}$ CNRS, Laboratoire Charles Coulomb, UMR 5521, F-34095 Montpellier Cedex 5, France \\ ${ }^{3}$ Université Paris Diderot, Sorbonne Paris Cité, Laboratoire Matière et Systèmes Complexes (MSC), \\ UMR 7057 CNRS, F-75205 Paris, France
}

(Received 27 December 2012; published 2 August 2013)

\begin{abstract}
We report theoretical predictions and measurements of the capillary force acting on a spherical colloid smaller than the capillary length that is placed on a curved fluid interface of arbitrary shape. By coupling direct imaging and interferometry, we are able to measure the in situ colloid contact angle and to correlate its position with respect to the interface curvature. Extremely tiny capillary forces down to femtonewtons can be measured with this method. Measurements agree well with a theory relating the capillary force to the gradient of Gaussian curvature and to the mean curvature of the interface prior to colloidal deposition. Numerical calculations corroborate these results.
\end{abstract}

DOI: 10.1103/PhysRevLett.111.058302

PACS numbers: 82.70.Dd, 68.03.Cd

Strong normal restoring forces due to the surface tension are sufficient to confine solid objects at fluid interfaces. This trapping can be cleverly used to both address fundamental problems [1,2] and to create new materials [3,4]. In many studies, the interface is planar and spatially uniform, merely providing a $2 \mathrm{D}$ confinement on particles. When curved, interfaces might play a more active role, imposing a lateral force on the particles. Usually, the curvature is induced by the colloids themselves. This is the case of heavy colloids that attract each other by falling in the gravitational well they have created [5]. When the effective weight of the trapped particles is negligible, a nonspherical shape [6] or the pinning of the triple line [7-9] induces a surface deformation. Lateral forces may emerge from the coupling with such capillary induced curvature of the interface [10]. A spectacular demonstration of this force is the meniscus-climbing technique of the beetle larva [11]. In the presence of a curved interface, the mechanical equilibrium conditions at the triple line larva-water-air impose an extra surface deflection and thus a lateral force on the larva. This force is also sufficient to drive micronlong cylinder self-assembly on a water-air curved interface [12]. Despite these interesting studies, a full comparison between experiment and theory is still lacking. Theories are restricted to spherical colloids trapped on a minimal surface [13] or with weak mean curvature [14], and no effects on spherical colloids have been reported so far.

In this Letter, we combine theory and experiment to address the capillary force acting on a spherical colloid placed on a curved fluid interface. We develop a new theoretical model able to predict this force in the general case of interfaces with arbitrary curvature. Using a built-in interferometric method coupled with particle tracking, we measured the femtonewton forces which control the equilibrium position of microspheres on a curved interface. We found a good agreement with our theoretical predictions.

Let us consider a spherical colloid of radius $a$ trapped at an interface of tension $\gamma_{\mathrm{LV}} \equiv \gamma$ between a liquid L and a vapor $\mathrm{V}$. At equilibrium, the contact angle between the interface and the colloid (C) surface is everywhere equal to the Young angle $\theta$ with $\gamma \cos \theta=\left(\gamma_{\mathrm{CV}}-\gamma_{\mathrm{CL}}\right)$ [15]. The interface prior to colloid deposition is assumed to be arbitrarily deformed with typical curvatures much smaller than $a^{-1}$. If $a$ is much smaller than the capillary length [15], the gravitational corrections to the capillary force can be neglected [16,17]. The tangential force $f$ acting on the colloid can then be expressed as the gradient of a scalar potential $W$, which is expected to depend only on the local curvature tensor field prior to colloid deposition $K_{i j}(\boldsymbol{r})=\boldsymbol{n} \cdot D_{i} \boldsymbol{t}_{j}$, where $i, j \in\{1,2\}, \boldsymbol{n}$ is the normal to the interface, $\left(\boldsymbol{t}_{1}, \boldsymbol{t}_{2}\right)$ is a tangential basis, and $D_{i}$ is the covariant derivative [18]. We expand $W$ in terms of all the scalars that can be formed by contracting the tensors $K_{j k}$ (order 1$), K_{j k} K_{\ell m}$ (order 2), $D_{j} D_{k} K_{\ell m}$ and $K_{j k} K_{\ell m} K_{n p}$ (order 3), etc. Laplace law [15] imposes the total curvature $K_{j}^{j}$ to be constant (Einstein's summation convention used). Then, taking into account that $D_{i} K_{j k}=D_{j} K_{i k}$ [18], the force $f_{i}=-D_{i} W$ reduces to $f_{i} \propto\left(\lambda+\mu K_{\ell}^{\ell}\right) D_{i}\left(K_{j}^{k} K_{k}^{j}\right)+$ $O(5)$. In terms of the principal curvatures $c_{1}$ and $c_{2}$ of the interface, $K_{\ell}^{\ell}=c_{1}+c_{2}$ and $K_{j}^{k} K_{k}^{j}=\left(K_{\ell}^{\ell}\right)^{2}-2 c_{1} c_{2}$, and thus

$$
f_{i}=\left[\lambda+\mu\left(c_{1}+c_{2}\right)\right] D_{i}\left(c_{1} c_{2}\right)+O(5),
$$

where the constants $\lambda$ and $\mu$ depend only on $\gamma, a$, and $\theta$.

To determine $\lambda$ and $\mu$, we calculate the interface shape in the presence of the colloid for an arbitrary asymptotic 
profile. The system free energy is $\mathcal{F}=\gamma \mathcal{A}_{\mathrm{LV}}+$ $\gamma_{\mathrm{CL}} \mathcal{A}_{\mathrm{CL}}+\gamma_{\mathrm{CV}} \mathcal{A}_{\mathrm{CV}}+P \mathcal{V}_{\mathrm{L}}$, with $\mathcal{A}_{\mathrm{XY}}$ the area of the $\mathrm{X}-\mathrm{Y}$ interface, $\mathcal{V}_{\mathrm{L}}$ the volume of $\mathrm{L}$, and $P$ the pressure jump across the L-V interface (see the Supplemental Material [19]). For small deformations of the latter,

$$
\begin{aligned}
\mathcal{F} \simeq & \int_{\bar{S}}\left[\gamma\left(1+\frac{1}{2}(\boldsymbol{\nabla} u)^{2}\right)+P u(\boldsymbol{r})\right] d \boldsymbol{r} \\
& +\int_{S}\left[\gamma \cos \theta \sqrt{1+(\boldsymbol{\nabla} h)^{2}}+P[H+h(\boldsymbol{r})]\right] d \boldsymbol{r},
\end{aligned}
$$

up to a constant. Here, $u(\boldsymbol{r})$ describes in the Monge gauge the height of the interface above a reference plane parametrized by $\boldsymbol{r}, H$ is the height of the center of the colloid, and $h(\boldsymbol{r})$ is the height of the colloid with respect to its center (Fig. 1). The domain $S$ is the projection of the upper cap on the reference plane and $\bar{S}$ the rest of this plane. The equilibrium configuration is obtained by minimizing $\mathcal{F}$ with respect to arbitrary variations of $u(\boldsymbol{r}), H$, and the boundary of $S$, parametrized by $r=\rho(\phi)$ in polar coordinates. As a result, we obtain the Laplace law $\gamma \nabla^{2} u=P$, the equilibrium conditions of the contact line

$$
\begin{aligned}
1+ & \frac{1}{2}(\nabla u)^{2}-\cos \theta \sqrt{1+(\nabla h)^{2}} \\
& +\left(\frac{1}{\rho^{2}} \rho_{\phi} u_{\phi}-u_{r}\right)\left(u_{r}-h_{r}\right)=0,
\end{aligned}
$$

and of the vertical position of the colloid

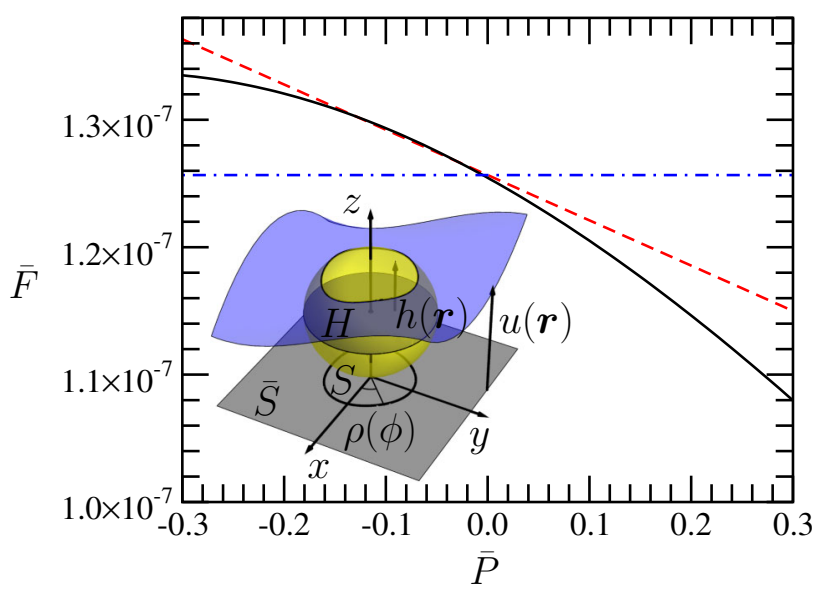

FIG. 1 (color online). Normalized force $\bar{F}=f_{x} /(\gamma L)$ vs normalized pressure $\bar{P}=P L / \gamma$ for a colloid of radius $a=0.2 L$ with Young angle $\theta=45^{\circ}$. The boundary deformation corresponds to $u_{2}=u_{3}=5 \times 10^{-3} L$, which yields, in the absence of the colloid, a Gaussian curvature gradient $\nabla\left(c_{1} c_{2}\right) \simeq-6 \times$ $10^{-4} L^{-3}$. The solid (black) line is the exact numerical result, the dashed (red) line is our analytical result (7), and the dash-dotted (blue) line corresponds to the analytical result without the pressure (i.e., total curvature $c_{1}+c_{2}$ ) correction [13]. The inset shows the parameters defining the geometry of the spherical colloid trapped at a liquid interface.

$$
\int_{0}^{2 \pi}\left(\frac{1}{\rho} \rho_{\phi} u_{\phi}-\rho u_{r}+\frac{P}{2 \gamma} \rho^{2}\right) d \phi=0,
$$

plus the matching condition $u[\rho(\phi), \phi]=H+h[\rho(\phi), \phi]$. In the above formulas, the subscripts indicate partial derivatives, e.g., $u_{r}=\partial u / \partial r$.

To deal with the free boundary problem at the contact line, we search for a perturbative solution $u(r, \phi)=u_{0}(r)+$ $\epsilon u_{1}(r, \phi), \rho(\phi)=\rho_{0}+\epsilon \rho_{1}(\phi)$, and $H=H_{0}+\epsilon H_{1}$, where $\epsilon$ is a small parameter and the zeroth-order solution $u_{0}$ is a parabola (constant curvature accounting for $P$ ). Inserting this perturbative expansion into the equilibrium equations yields $\nabla^{2} u_{1}=0$ with, at first order in $\epsilon$, boundary conditions on the fixed, unperturbed contour $r=\rho_{0}$. The most general solution for $u_{1}$ breaking the axial symmetry can be expanded in multipoles as

$$
\begin{aligned}
\bar{u}_{1}(r, \phi)= & \sum_{n=1}^{\infty}\left(A_{n} \bar{r}^{n}+A_{n}^{\prime} \bar{r}^{-n}\right) \cos (n \phi) \\
& +\sum_{n=1}^{\infty}\left(B_{n} \bar{r}^{n}+B_{n}^{\prime} \bar{r}^{-n}\right) \sin (n \phi),
\end{aligned}
$$

where $\bar{u}=u / \rho_{0}$ and $\bar{r}=r / \rho_{0}$. Note that $A_{n}$ and $B_{n}$ characterize the asymptotic profile. Matching the boundary conditions yields, at first order in $p=P a / \gamma$ (i.e., for $c_{1}+$ $\left.c_{2} \ll a^{-1}\right), A_{n}^{\prime} / A_{n}=B_{n}^{\prime} / B_{n}=(n-1) /(n+1)+O\left(p^{2}\right)$. Integrating the stress tensor [20] (I is the unit tensor)

$$
\Sigma=\left[\gamma\left(1+\frac{1}{2}(\nabla u)^{2}\right)+P u\right] \mathbf{I}-\gamma \nabla u \otimes \nabla u
$$

on an arbitrary contour surrounding the colloid yields a curvature force parallel to the reference plane

$\boldsymbol{F}_{c}=-\frac{\pi}{6} \gamma a^{4} \sin ^{4} \theta\left[1-2 a\left(c_{1}+c_{2}\right) \cos \theta\right] \nabla\left(c_{1} c_{2}\right)$,

which agrees with Eq. (1). Here, $\boldsymbol{\nabla}\left(c_{1} c_{2}\right)$ is the gradient of the Gaussian curvature, prior to colloid deposition, at the position where it will be placed. It is thus calculated on the unperturbed interface and depends only on the coefficients $A_{2}, A_{3}, B_{2}$, and $B_{3}$. Equation (7) generalizes the result of Würger [13] to surfaces of arbitrary shapes. To validate our theoretical formula (7), we consider a spherical colloid free to slide along the $z$ axis. Imposing a deformation $u(L, \phi)=u_{2} \cos (2 \phi)+u_{3} \cos (3 \phi)$ far from the colloid, we calculate numerically the resulting shape $u(r, \phi)$ of the interface and the force $f_{x}$ exerted on the colloid [17]. We find a good agreement, as shown in Fig. 1.

The value of the expected capillary force obtained from Eq. (7) is extremely weak. Indeed, typical values of surface tensions at fluid-air interfaces $\gamma \approx 50 \mathrm{mN} / \mathrm{m}$, particle radius $a=1 \mu \mathrm{m} \ll \ell_{c}$, and gradients of Gaussian curvature of the order of $1 / R^{3} \approx 10^{12} \mathrm{~m}^{-3}$, where $a \ll R=$ $100 \mu \mathrm{m}$, give a force of the order of femtonewtons. Measuring such tiny forces to test the experimental validity of Eq. (7) is rather challenging [21,22]. We use a null force method where the known gravity force on the particle 
competes with the force $\boldsymbol{F}_{c}$ to be measured. Especially devised setup and method, combining direct imaging of the particle and interferometry, give the capillary force down to the femtonewton scale.

In the experiment, we use designed containers formed by two coaxial cylinders in which the liquid filling the gap exhibits an interface with a spontaneous large gradient of Gaussian curvature. Using standard photolithography procedures, we patterned rings of a negative photoresist (SU8-2025, Microchem) on a Si wafer. After polydimethylsiloxane (PDMS) molding, we obtained $100 \mu \mathrm{m}$ thick cuvettes of inner and outer radii $R_{1}=100 \mu \mathrm{m}$ and $R_{2}=200 \mu \mathrm{m}$ (Fig. 2). The cuvettes are filled with mineral oil (M3516, Sigma).

The air-oil interface is accurately characterized by means of a custom-made phase shift interferometer (PSI) mounted on a Leica 2500 optical microscope. The cuvettes are fixed on the stage of the microscope placed on a vibration-isolation table (CVI). A $633 \mathrm{~nm}$ monochromatic light and a $\times 25$ Mirau objective (Nikon) are used to create interferences between the reflected beams at the reference mirror and at the air-oil interface. The interferometer is first vertically aligned by maximizing the fringes width at a horizontal air-glycerin interface. With the cuvette, a set of interference images is collected by a CCD Sony XCDX710 camera during the vertical scanning of the objective over distances of a wavelength using a nanopositioner (Nano-F, MCL). The continuous interface profile is then reconstructed by standard phase-unwrapping algorithms. It follows the Young-Laplace equation $P=\gamma\left(c_{1}+c_{2}\right)$ that can be worked out analytically because hydrostatic
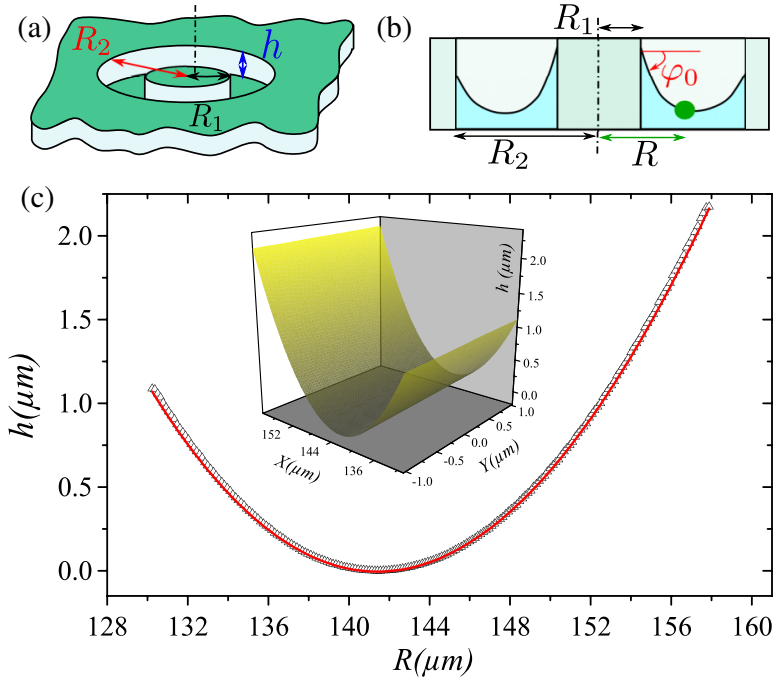

FIG. 2 (color online). Experimental setup and geometric notations in (a) 3D view and (b) side view. (c) Example of an experimental radial profile with its best fit (plain curve) obtained from Eq. (8) and yielding $\varphi_{0} \approx 57^{\circ}$. Inset: Interface region reconstructed by PSI from which the radial profile has been extracted. pressure is negligible at the considered scale. Using notations of Fig. 2(b), the slope angle $\varphi(R)$ is given by

$$
\sin \varphi(R)=\frac{\sin \varphi_{0}}{R_{2}-R_{1}}\left(R-\frac{R_{2} R_{1}}{R}\right),
$$

where $\varphi_{0}$ is the corresponding value at the container walls, fixed by the Young's relation at the air-oil-PDMS triple line. The bottom of the interface should be located at the radius $R_{g}=\sqrt{R_{1} R_{2}} \approx 141 \mu \mathrm{m}$, independently of $\varphi_{0}$. The fringes are indeed centered at $R_{g}$. Moreover, the PSI reveals that Eq. (8) well describes the actual profile [see Fig. 2(c)], with best-fitting values $\varphi_{0}=53^{\circ} \pm 5^{\circ}$ over different samples, corresponding to the measured contact angle of a mineral oil droplet deposited on PDMS.

Silica beads of $3.93 \pm 0.44 \mu \mathrm{m}$ diameter (Bangs Laboratories) have been used. Their contact angle has been controlled by treating their surface either with oleophilic N,Ndimethyl-N-octadecy-3-aminopropyltrimethoxysilylchloride (DMOAP) [23] or with oleophobic perfluorooctyltrichlorosilane (PFOTS). After treatment, the colloids are thoroughly dried and deposited individually with an air pulse at the air-oil interface. A bead rapidly sits in the vicinity of $R_{g}$ due to its higher density ( $\rho_{b} \approx 2 \mathrm{~g} \mathrm{~cm}^{-3}$ compared to $\rho_{o}=0.84 \mathrm{~g} \mathrm{~cm}^{-3}$ for oil). This localization allows the in situ measurement of $\theta$ [23] by vertical scanning interferometry (VSI) [24] achieved with the PSI setup (see the Supplemental Material [19]). We found $\theta=0^{\circ}-5^{\circ}$ for DMAOP treated beads and $\theta=30^{\circ} \pm 2^{\circ}$ for PFOTS ones.

Once deposited, a colloid can be moved across the interface with a tiny air jet. The relaxation dynamics to its equilibrium position is recorded at $30 \mathrm{fps}$. From image analysis, we extract data sets of the particle center of mass. A typical trajectory is shown in Fig. 3(a) for a $0^{\circ}$ contact angle bead. Starting from the vicinity of the outer radius, the particle falls down toward $R_{g}$, the minimum height of the profile. This behavior is expected since only gravity and buoyancy forces act. For oleophobic beads, a different behavior is observed. As shown in Fig. 3(b), a bead partially embedded in oil overpasses its gravity potential minimum, climbs the slope, and finally reaches an equilibrium radius $R_{\text {eq }}$ such than $R_{1}<R_{\text {eq }}<R_{g}$. In Fig. 3(c), different experiments on the same bead and on different beads and cuvettes are presented. A systematic radial deviation from the minimum height location $R_{g}-R_{\text {eq }}=$ $3.6 \pm 0.9 \mu \mathrm{m}$ is observed for PFOTS treated beads [see Fig. 3(c)]. It indicates the presence of an additional inward radial force. The dispersion of points around $R_{\text {eq }}$ for different cuvettes and beads is roughly the same as the one for the different experiments repeated on the same bead. It thus may be related more to differences in the pinning of the triple line on the bead from one experiment to another rather than to the small differences on beads sizes and cuvettes.

More quantitatively, the interface profile from Eq. (8) yields, with Eq. (7), a curvature force, 

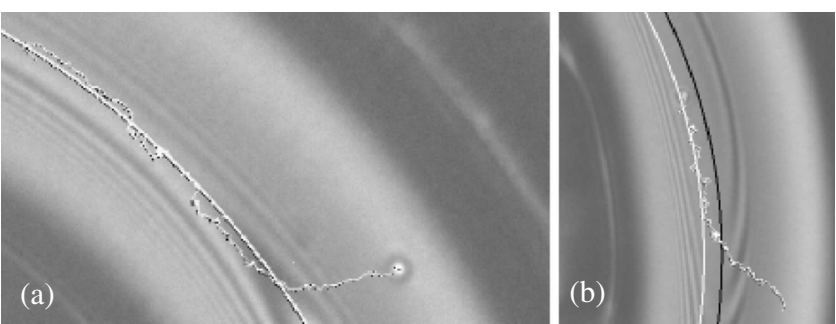

(c)

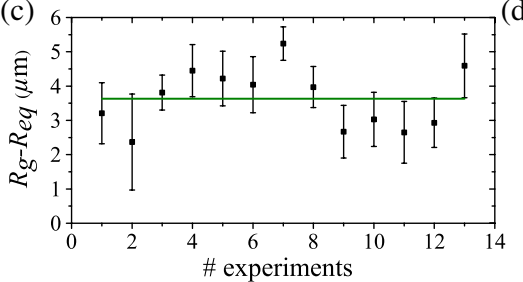

(d)

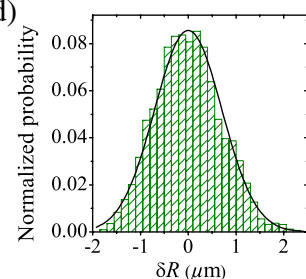

(e)

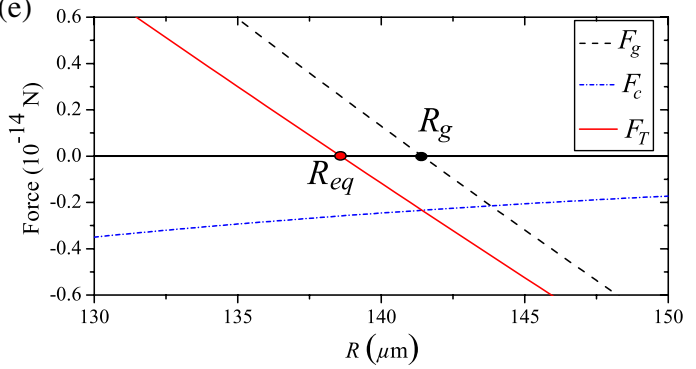

FIG. 3 (color online). Example of trajectories of beads on the air-oil interface with contact angle (a) $\theta=0^{\circ}$ and (b) $\theta=30^{\circ}$. The black line is the locus of the radial minimum height of the interface $\left(R_{g}\right)$. The white circle corresponds to the final equilibrium radius $R_{\text {eq }}$ of the bead. Note also the visible thermally driven fluctuations $\delta R$ around $R_{\text {eq }}$ (c) Deviation $R_{g}-R_{\text {eq }}$ for various experiments made with different beads with $\theta=30^{\circ} \pm 2^{\circ}$ and cuvettes with $\left\langle\delta R^{2}\right\rangle$ error bars. (d) Typical probability distribution function vs $\delta R$ and best fit with a normal distribution. (e) Curvature force $F_{c}$, gravity force $F_{g}$, and the resulting total force $F_{T}$ vs $R . F_{c}$ and $F_{g}$ are plotted using $\gamma=28 \mathrm{mN} \mathrm{m}^{-1}$ [25], $\phi_{0}=53^{\circ}, \theta=30^{\circ}, R_{1}=100 \mu \mathrm{m}, R_{2}=200 \mu \mathrm{m}, a=2 \mu \mathrm{m}$, and $m_{f}=5.75 \times 10^{-11} \mathrm{~g}$.

$F_{c}=-\frac{2 \pi \gamma a^{4} \sin ^{4} \theta \sin ^{2} \varphi_{0}}{3\left(R_{2}^{-1}-R_{1}^{-1}\right)^{2}}\left(1-\frac{4 a \sin \varphi_{0} \cos \theta}{R_{2}-R_{1}}\right) \frac{\cos \varphi(R)}{R^{5}}$.

This force is negative and varies only little around $R_{g}$ [see Fig. 3(e)] and should be counterbalanced by the bead effective weight (gravity and buoyancy) $F_{g}=-m_{f} g \sin \varphi(R)$, where the effective mass $m_{f}=\pi a^{3} \rho_{o}\left[4 \rho_{b} / \rho_{o}-2-\right.$ $\left.3 \cos \theta+\cos ^{3} \theta\right] / 3$ is obtained from geometric considerations.

The weight can therefore be used to determine the curvature force. We first directly measure $m_{f}$ by considering the thermally driven fluctuations $\delta R(t)$ around $R_{\text {eq }}$ [see Figs. 3(a) and 3(b)]. At first order, thermal energy competes only with the gravitational potential since $F_{c}$ is nearly constant near $R_{g}$. The linearized restoring force $f$ acting on the bead located at $R_{\mathrm{eq}}+\delta R$ is then $f(\delta R) \approx$ $-2 m_{f} g \sin \varphi_{0} /\left(R_{2}-R_{1}\right) \delta R$, yielding a Boltzmann distribution probability $P(\delta R) \propto \exp \left[-(1 / 2) \delta R^{2} / w^{2}\right]$, where $w^{2}=k_{B} T\left(R_{2}-R_{1}\right) /\left(2 m_{f} g \sin \varphi_{0}\right)$. The measured distribution probability is well fitted by this function. For the example shown Fig. 3(d), the standard deviation $w \approx 0.67 \pm 0.03 \mu \mathrm{m}$ yields $m_{f} \approx 5.7 \pm 0.5 \times 10^{-11} \mathrm{~g}$ and a force $F_{c}=-2.5 \pm 0.3 \mathrm{fN}$. Corresponding calculated forces $F_{g}, F_{c}$, and $F_{T}=F_{c}+F_{g}$ vs $R$ are summarized in Fig. 3(e). The new equilibrium position $R_{\text {eq }}$ is expected to be shifted by $-3 \mu \mathrm{m}$ with respect to the one imposed by the gravity alone $R_{g}$. This is in good agreement with the measurements. Note that our experimental resolution does not allow us to check directly the mean-curvature correction in Eq. (7). Indeed, we have $c_{1}+c_{2}=2 \sin \varphi_{0} /$ $\left(R_{2}-R_{1}\right) \simeq 0.016 \mu \mathrm{m}^{-1} \ll a^{-1}$. However, we have verified the latter by using numerical calculations (cf. Fig. 1).

In this Letter, we investigated both theoretically and experimentally the role of the gradient of curvature on the lateral force acting on microspheres confined at a fluid interface of arbitrary shape. By using a new theoretical approach, based on a direct calculation of the forces in an arbitrary imposed interface morphology, we showed that the force acting on the colloid should depend both on the gradient of Gaussian curvature and on the mean curvature. The experimental measurement of this capillary force was challenging due to its extremely weak value of the order of femtonewtons. We devised an experimental setup based on a null force method coupling particle tracking and interferometry to access to such tiny forces. The measured force agrees with our theoretical predictions. Our findings open the way to the control of the force and the assembly of micrometric size particles by the design of the interface morphology. We believe also that the used optical method and setup can be applied in other contexts and offer a new method to increase the sensitivity of force measurement experiments down to the femtonewton scale.

The authors acknowledge financial support from the French Agence Nationale de la Recherche (Contract No. ANR-07-BLAN-0243-SURFOIDS).

[1] K. Zahn, R. Lenke, and G. Maret, Phys. Rev. Lett. 82, 2721 (1999).

[2] V. N. Manoharan, M. T. Elsesser, and D. J. Pine, Science 301, 483 (2003).

[3] A.D. Dinsmore, M.F. Hsu, M.G. Nikolaides, M. Marquez, A. R. Bausch, and D. A. Weitz, Science 298, 1006 (2002).

[4] K. Stratford, R. Adhikari, I. Pagonabarraga, J.-C. Desplat, and M. E. Cates, Science 309, 2198 (2005).

[5] M. M. Nicolson, Proc. Cambridge Philos. Soc. 45, 288 (1949).

[6] J.C. Loudet, A. M. Alsayed, J. Zhang, and A. G. Yodh, Phys. Rev. Lett. 94, 018301 (2005).

[7] D. M. Kaz, R. McGorty, M. Mani, M. P. Brenner, and V. N. Manoharan, Nat. Mater. 11, 138 (2011). 
[8] D. Stamou, C. Duschl, and D. Johannsmann, Phys. Rev. E 62, 5263 (2000).

[9] B. J. Park and E. M. Furst, Soft Matter 7, 7676 (2011).

[10] M. Oettel, A. Domínguez, and S. Dietrich, Phys. Rev. E 71, 051401 (2005).

[11] D. L. Hu and J. W. M. Bush, Nature (London) 437, 733 (2005).

[12] M. Cavallaro, L. Botto, E. P. Lewandowski, M. Wang, and K. J. Stebe, Proc. Natl. Acad. Sci. U.S.A. 108, 20923 (2011).

[13] A. Würger, Phys. Rev. E 74, 041402 (2006).

[14] C. Zeng, F. Brau, B. Davidovitch, and A. D. Dinsmore, Soft Matter 8, 8582 (2012).

[15] L. D. Landau, Fluid Mechanics (Pergamon, London, 1959).

[16] P. A. Kralchevsky, V. N. Paunov, N.D. Denkov, and K. Nagayama, J. Colloid Interface Sci. 167, 47 (1994).

[17] P. Galatola and J. Fournier (to be published).
[18] F. David, Geometry and Field Theory of Random Surfaces and Membranes (World Scientific, Singapore, 1989).

[19] See Supplemental Material at http://link.aps.org/ supplemental/10.1103/PhysRevLett.111.058302 for details on the theoretical model and the interferometric technique to measure the contact angle.

[20] J.-B. Fournier, Soft Matter 3, 883 (2007).

[21] L.E. Helseth and T. M. Fischer, Phys. Rev. E 68, 051403 (2003).

[22] L.E. Helseth, R. Muruganathan, Y. Zhang, and T.M. Fischer, Langmuir 21, 7271 (2005).

[23] M. A. Gharbi, M. Nobili, M. In, G. Prevot, P. Galatola, J.-B. Fournier, and C. Blanc, Soft Matter 7, 1467 (2011).

[24] K. Larkin, J. Opt. Soc. Am. A 13, 832 (1996).

[25] B. J. Mullins, A. Pfrang, R. D. Braddock, T. Schimmel, and G. Kasper, J. Colloid Interface Sci. 312, 333 (2007). 